\title{
JUEGOS EN LA ENSEÑANZA \\ DE LA INGENIERÍA DEL SOFTWARE
}

\author{
Diego Guerrero Peña ${ }^{1}$ \\ Helmuth Trefftz Gómez ${ }^{2}$ \\ RAQUEL ANAYA ${ }^{3}$
}

\section{Resumen}

Los juegos serios, en particular los videojuegos, han aumentado su uso en la enseñanza de la ingeniería del software como elementos motivacionales para el aprendizaje significativo.

Este artículo resalta la existencia o carencia de los soportes pedagógicos y didácticos que los sustentan mediante el análisis derivado del estado del arte correspondiente a los juegos serios respecto a la Ingeniería de Software; además, se proponen postulados pedagógicos que los sustentan y una clasificación de los mismos.

Los resultados que aquí se presentan hacen parte de una investigación desarrollada en el Instituto Tecnológico Metropolitano (ITM) como parte del proyecto de investigación de la Maestría en Ingeniería Informática de la Universidad Eafit que está llevando a cabo el profesor Diego Alejandro Guerrero Peña.

1 Diego Alejandro Guerrero Peña, Ingeniero de sistemas, Especialista en Gerencia de Proyectos, candidato a Magíster en Ingeniería Informática de Eafit, Profesor Auxiliar del ITM. diegoguerrero@itm.edu.co

2 Helmuth Treffz Gómez, Ingeniero de Sistemas, Magister en Masters Of Science In Computer Science.Doctorado en Electrical And Compuer Engineering, Doctorado en Electrical And Compuer Engineering y Profesor Titular Universidad Eafit. htre®tz@Eafit.edu.co

3 Raquel Anaya de Paez, Ingeniero de sistemas, Doctorado en Ingeniería de la Programación e Inteligencia Artificial, Universidad Politécnica de Valencia, España. Profesor Titular Universidad Eafit ranaya@Eafit.edu.co. 


\section{Palabras claves}

Juegos Serios, TICs, educación, aprendizaje, ingeniería del software.

\section{Abstract}

Serious games, mainly videogames, have increased their use in the teaching of software engineering as motivation factors for the significant learning. This paper stresses on the existence or lack of their pedagogical and didactic supports, through the analysis derived from the state of the art of the serious games related to the software engineering. Furthermore, this work proposes pedagogical postulates that bear these games and makes a classification of them. The results shown here make part of a research developed under the projects: Information and Communication Technologies as a Support for Teaching (TICAD for its acronym in Spanish) of the InstiTuTo TECnológico Metropolitano (ITM) and Information and Communication Technologies used for software engineering teaching (TICSOFT for its acronym in Spanish) of Masters in Computer Engineering of the Eafit University.

\section{Keywords}

Serious games, TIC's, education, learning, software engineering. 


\section{INTRODUCCIÓN}

Las nuevas Tecnologías de la Información y las Comunicaciones (TICs): blogs, foros virtuales, plataformas de aprendizaje electrónico (elearning), videochats, videojuegos, entre otros, han evolucionado hasta el punto de facilitar el uso de juegos para la enseñanza, i.e., una serie de estrategias que utilizan los juegos como mecanismo de apoyo a las didácticas de los docentes para la enseñanza de determinados temas de las asignaturas (Urrego \& Castaño, 1999)

Existen varias asociaciones virtuales, empresas desarrolladoras de software, investigadores, expertos en el tema, proveedores de tecnología (Ward, 2008), estudiantes experimentadores y docentes que tratan el tema de los Juegos Serios, como el docente Michael Zyda ${ }^{1}$ (Zyda, 2007), Serios Games (Houk\& Ibraim, 2008; Feature Making, 2008; Alhadeff, 2008), entre otros, pero solo algunos analizan el aprendizaje que pueden alcanzar los alumnos mediante el uso de los juegos. La mayoría de las asociaciones no manejan mayor rigurosidad en los resultados obtenidos y otras carecen de los mismos. De igual manera, hay ausencia de estándares para la evaluación y clasificación de los juegos, y no hay soportes pedagógicos sustentables que las soporten, en la mayoría de los casos.

Las estrategias de enseñanza aprendizaje requieren de acompañamiento y supervisión del docente, ya que los juegos por sí solos, al igual que otras estrategias didácticas, no funcionan adecuadamente, es el docente quien debe enseñar y facilitar al estudiante descubrir la lógica del conocimiento objeto de aprendizaje (Bass, 1999) de forma amena y agradable, y quien debe seleccionar la estrategia más apropiada que permita una retención larga y

1 Michael Zyda (zyda@usc.edu) director de GamePipe Laboratory, professor de práctica de ingeniería el departamento de Ciencias de la Computación y miembro asesor del Instituto de Ciencias de la Información de la Universidad de Southern California, Los Angeles. http://gamepipe.usc. edu/ zyda/pubs/CACM-July2007.pdf 
una memorización eficaz, de ahí la importancia del uso de las TICs como elementos facilitadores, y particularmente los juegos serios, juegos que, siendo divertidos, facilitan el aprendizaje en un área específica del conocimiento, éstos utilizan un entorno lúdico para alcanzar objetivos como el de comunicar, educar o entrenar. Para un mejor estudio y análisis de los juegos serios se deben clasificar y sustentar pedagógicamente para asegurar que generan aprendizaje significativo.

En el presente artículo se plantearán elementos pedagógicos y didácticos como postulados pedagógicos que sustentan la propuesta de clasificación y uso de los juegos serios en los procesos de enseñanza aprendizaje, igualmente el levantamiento del estado del arte de los juegos para la enseñanza, especificando aspectos en la enseñanza de la ingeniería del software (Anaya, 2006) y teniendo en cuenta el Modelo para la Enseñanza de la Ingeniería del Software (Guide to the software engineering body of knoledge) SWEBOK de la IEEE.

\section{MATERIALES Y MÉTODOS}

La información sobre juegos serios se obtiene principalmente de la literatura reciente, pero debido a su escasez también se recoge información de diferentes fuentes de empresas desarrolladoras de juegos serios, en sitios de libre acceso en la Web. El trabajo se centra en los juegos para la enseñanza a nivel de educación superior en todo el mundo, y específicamente en la enseñanza de la ingeniería del software, aunque se analizan algunos trabajos realizados para la enseñanza de las ciencias básicas entre otros, en los niveles de educación superior, primaria y secundaria, dada la importancia en su futura utilización en la enseñanza de la ingeniería del software.

También se toman trabajos pedagógicos aplicados a las TICs para la enseñanza y autores en pedagogía y didáctica muy relevantes de donde se obtienen los postulados pedagógicos analizados y propuestos (Trujillo \& Jaramillo, 2006, pág. 98; Guerrero, 2007) y (Colon, 2002) entre otros. 
Algunos de los análisis estadísticos son resultados de experimentos realizados por docentes en diferentes cursos. Las respuestas obtenidas se clasifican con base en el modelo de las 4P's (Pressman, 2000), que incluye las dimensiones. Personal, Proceso, Proyecto y Producto.

\section{Consideraciones generales}

\section{A. Posiciones contrarias en el uso de los juegos}

No siempre se han considerado los juegos como un apoyo en el aprendizaje, en esta investigación se encontraron diferentes posiciones, unas que apoyan los juegos, otras, detractores que opinan que solo permiten un aprendizaje memorístico de corto tiempo, caso puntual el artículo "Can Games Be Used to Teach?" (Peshette \& Thornburg, 2006). Este exhibe puntos de vista con respecto a la utilización de los juegos para generar en el estudiante aprendizaje significativo. Mas el artículo no precisa resultados cuantificables que demuestren sus conclusiones.

\section{B. Inversiones financieras en juegos para la enseñanza}

Según Javier Carbonel, (2008) el mercado actual de los juegos dirigidos a algo más que diversión, es de 150 millones de dólares y en el año 2011 será de 1 billón de dólares. Según la predicciones para el 2007 de la Oficina de Administración del Presupuesto de los Estados Unidos (Carless, 2007) la industria de los videojuegos debió aumentar alrededor de $\$ 60$ mil millones en ingresos para el 2007 (U.S. Office of Management and Budget, 2004) casi el tamaño de los gastos en investigación, desarrollo, ensayo y evaluación del Departamento de Defensa EE.UU. Así mismo, existen algunos blogs dedicados como ExeBlog "desarrollo de videojuegos y más" (Blázquez, 2008) y empresas como NINTENDO NINTENDO, (2008), Intelligent Windows Management (intelligent windows management, 2008) con millonarias inversiones. 


\section{IV.Resultados}

\section{Postulados Pedagógicos}

A continuación se presentan, de manera sintetizada y como postulados, los principios pedagógicos que sustentan en gran parte, el uso de los juegos serios:

Postulado 1. La mediación efectuada por las TIC genera múltiples factores estructurales que rodean e influyen en la relación didáctica que se da entre el docente y el estudiante, y entre, éstos y el saber. La tecnología se constituye como una instancia para apoyar situaciones de gestión de clausuras explícitas o implícitas, que evolucionan en el mantenimiento constante del contrato de deberes y derechos entre quien enseña y quien aprende, acciones que se espera sirvan de ayuda para lograr una construcción más autónoma por parte del alumno (Trujillo \& Jaramillo, 2006:98).

En el Modelo Por Competencias, el tiempo independiente es considerado fundamental para el trabajo de los estudiantes fuera de clase, por tal razón, utilizar las TIC, en este caso los Juegos Serios, son de vital importancia, puesto que facilitan y potencializan la aprehensión del conocimiento y el acercamiento al saber de forma amena y divertida.

Postulado 2. En una siguiente construcción de correlaciones se reconocen las diferentes intervenciones que los estudiantes hacen con la tecnología para interactuar con el docente, sus compañeros y los contenidos, o hacer despliegue de sus propuestas ante los otros. En estos escenarios se marcan las diferencias entre la unidireccionalidad y la bidireccionalidad, la sincronía y la asincronía, la conectividad y la no conectividad a la red. Todas estas situaciones potencian procesos de apropiación, proyección, extrañamiento y reconocimiento alrededor del logro de expectativas individuales o colectivas, dentro o fuera de la intencionalidad curricular. Trujillo \& Jaramillo, (2006).

En los juegos la participación grupal es esencial, bajo la dirección del docente, los alumnos presentan propuestas de solución 
a problemas planteados, además las exponen entre ellos mismos con el fin de compartir experiencias que permitan la apropiación y revisión del conocimiento adquirido por éstos,

Postulado 3. "Es el hombre el que debe enfrentarse a la realidad vacía; un hombre que es capaz de vivir sin ayudas, porque ya no las necesita. Un hombre que ante nada será el encargado de crear el mundo" Colon, (2002) ${ }^{2}$. Es el aprendiz el que debe construir sus propios conocimientos y el que es responsable de los mismos.

En los juegos cada alumno debe de generar sus propias soluciones ante situaciones inesperadas permitiéndole generar su propio conocimiento mediante procesos de abducción, generados a través del juego.

Postulado 4. El contexto natural de ubicación de la Teoría del Caos es la Complejidad, de tal manera que sin situaciones complejas no se daría caos; por lo tanto, hablar de teoría del caos es hablar de la COMPLEJIDAD; por lo tanto, supone aceptar el desorden, la innovación y el movimiento como aspectos inherentes a cualquier situación caótica, por lo que la teoría del caos, indudablemente, se nos presenta como otro fundamento para la Teoría Social y por ende para la Teoría Educativa[...] Colon, (2002).

El estudiante debe enfrentar la solución a una situación en particular (juego serio), que dé solución a un problema existente. Esta situación le permite explorar nuevos conocimientos y buscar cada vez información que le ayude a dar solución. También le implica trabajar en equipo y enfrentarse a diferentes situaciones de discusión, nuevas hipótesis, nuevas formas de trabajo, nuevos retos que superar, etc., que le permitirán reafirmar sus conocimientos y sus apreciaciones de los fenómenos observados.

Postulado 5. "La Teoría del Aprendizaje Significativo elaborada desde una posición Organicista que se centra en el aprendizaje producido por un contexto educativo, en una situación de interiorización o asimilación a través de la instrucción”. (Pozo, 1989, pág.209). De lo anterior, se extrae que el aprendizaje importante

2 Sólo se analiza la Teoría del caos- Superhombre de Nietzsche. 
que realiza un individuo, se adquiere por la relación de los nuevos conocimientos con los conocimientos previos.

La aplicación de la teoría del aprendizaje significativo permite al estudiante desarrollar la capacidad de relacionar los conceptos y conocimientos adquiridos con los nuevos y, poder generar con estas relaciones nuevos conocimientos. El juego serio permite realizar una correlación entre los conceptos aprendidos con los nuevos conceptos que se trabajan al interior del mismo. Igualmente, invita a que el docente en su didáctica les ayude a usar los conectores de una mejor manera.

Postulado 6. La razón como producto de interacción social ${ }^{3}$. Resaltar la coordinación de comunicaciones como valor fundamental para co-construir conocimiento. La comunicabilidad y la comprensión de eso que se comunica. La interacción social ejerce sobre los sujetos relaciones de expresión y de colaboración. A través de la confrontación con otros, el sujeto analiza su propio pensamiento y toma conciencia de su modo de organización, de tal manera que enriquece el mecanismo de la razón (Vygotski, 1996) y (Leontiev, 1981).

En los juegos se deben realizar estrategias las cuales cada grupo debe presentar a los otros, permitiendo la confrontación de las experiencias y el conocimiento adquirido; esto permite la creación de criterios sustentables de lo realizado como también el replanteamiento de conceptos adquiridos, lográndose una afirmación del saber en el estudiante.

D. Juegos aplicados directamente a la enseñanza de la ingeniería del software

En la Universidad Brown (Duvall et al, 2001), dos de los autores del artículo: Identifying Predictors of Success for an Objects First CS1 (Eger et al, 2006) junto con otros profesores adaptaron en los ejercicios de la asignatura: introducción a la programación los materiales para la enseñanza de los objetos en la programación orientada a objetos mediante gráficos. Los estudiantes crearon

3 Tomado de ANAYA y TRUJILLO, 2006. Principios Pedagógicos-Didácticos. En: Un modelo para la enseñanza en contexto de la Ingeniería de Software. 
programas diseñados para ilustrar los principios de composición y asociación.

La programación pedagogía multi-usuario para mejorar el estudio tradicional- M.U.P.P.E.T.S (Eger et al 2006; Bierre \& Phelps, 2003 y 2004), creó un ambiente colaborativo virtual para la enseñanza de la "introducción a la programación", éste permite a los estudiantes explorar la programación a través de la experimentación y la observación cuidadosa de causa efecto.

$\mathrm{Al}$ igual que otros artículos, en los dos anteriores no hay evaluaciones a los procesos donde se demuestre pedagógicamente las ventajas de la utilización de los juegos.

Otras investigaciones se han llevado a cabo en la Universidad Nacional de Colombia- Sede Medellín, el profesor Carlos Mario Zapata Jaramillo ha trabajado diferentes juegos para "Un curso Inicial de la Ingeniería del Software basado en Juegos" (Zapata, 2007) algunos de estos juegos se realizan de forma manual, mientras que otros son aplicaciones de software. En su artículo define siete juegos para estructurar un curso de ingeniería del software al igual que presenta los resultados obtenidos hasta el 2007.

Como conclusiones principales de estas investigaciones el profesor Carlos Zapata, expresa: algunos conceptos son más fáciles de explicar mediante juegos, ya que se constituyen en una estrategia didáctica, divertida, práctica y segura. El estudiante de Maestría en Ingeniería de Sistemas de la Universidad Nacional, y a la vez investigador de apoyo a la investigación formativa del ITM ${ }^{4}$, Carlos Ocampo dice que los juegos son una estrategia de enseñanza aprendizaje muy adecuada en algunos de los temas que el docente Carlos Zapata utiliza.

La técnica de evaluación utilizada fue: Encuestas con preguntas abiertas. Las respuestas se clasificaron con base en el modelo de las 4P's (Pressman, 2000), que incluye las siguientes dimensiones. Personal, Proceso, Proyecto y Producto

4 Instituto Tecnológico Metropolitano, institución universitaria ubicada en Medellín(Colombia). 
Esta es una de las investigaciones que se han realizado con mayor rigor, en lo que a juegos serios se refiere.

E. Clasificación de los juegos serios

Además de los juegos aplicados directamente a la enseñanza de la ingeniería del software, existen muchas clasificaciones creadas por diferentes autores y desarrolladores de juegos. La propuesta de clasificación parte de estos trabajos, indistintamente que sean los juegos virtuales o juegos físicos. Por respecto a sus autores, se indican las fuentes de donde fueron seleccionadas y se explican algunas clasificaciones, al igual en otras, se identifica su utilización en la enseñanza de la ingeniería del software.

Juego Documental (Diverted game): juegos enfocados a la educación sobre lugares, culturas, eventos históricos y políticos y algunos sobre turismo en general (Boiffard, 2008; Boiffard, 2008a; Under Ash, 2008; Unreal, 2008; Bordergames, 2008), ejemplos de empresas creadoras de este tipo de juegos es Blitz Games Studios (Blitz, 2008). Estos juegos pueden ser adaptados para la enseñanza de diferentes temas dentro de la ingeniería del software, asimilándolos a proyectos de desarrollo, donde los estudiantes representan diferentes roles como: Usuarios, directivos, analistas, desarrolladores etc.

Jugar para la Formación Específica (Edugame o Edumarket): Estos incluyen los utilizados para la enseñanza de la ingeniería del software, (Sebastien, 2008), incluye los juegos para el aprendizaje asistido, e.g. English Training (NINTENDo) para el aprendizaje del inglés, Pokemon PC Master (NINTENDO) para enseñar el manejo del PC a los más pequeños, Pokemon Learning League (NINTENDO) que ayuda a los docentes a reforzar conceptos básicos de matemáticas, lengua y ciencia de sus estudiantes, "Turn It All Off" (INTELLIGENT WINDOWS MANAGEMENT, 2008); también son muy utilizados en la enseñanza de las ciencias básicas (Gairín, 1990), José Gairín recoge resultados de investigaciones sobre los efectos que produce la utilización de juegos matemáticos en los alumnos. La importancia de este artículo radica en que estos resultados se pueden extender a la enseñanza de la ingeniería del software y usar para 
la conceptualización de los modelos de desarrollo de software, y temas similares. Es de aclarar que existen muchos juegos para la enseñanza de las ciencias básicas que de igual manera, pueden tener aplicación en algunos temas de ingeniería del software.

Juego sin Límite (Pervasive games): también conocidos como juegos omnipresentes (Vega, 2008) aprovechan las crecientes prestaciones de los aparatos tecnológicos y las conectividad para crear un entorno de juegos más allá del computador, con el mundo real como escenario y tantas posibilidades como permita la creatividad de los desarrolladores (Feature MaKing, 2008). Al igual que otros artículos y referencias, la sustentación desde la pedagogía y la didáctica es deficiente y no presentan evaluaciones que demuestren el nivel de aprendizaje de los alumnos.

Juegos Simultáneos en Tiempo Real (Serious Play) (Martinez \& Marquis, 2008): más que un juego es una metodología de juegos serios en tiempo real, el profesor permite que las personas que están en una actividad de Juegos Serios Play compartan los conocimientos, la experiencia y, sobre todo, las percepciones conscientes e inconscientes que tienen con respecto a lo que está ocurriendo en la organización. Aunque la apreciación es válida, Javier Martinez no hace una aclaración pedagógica sustentable, pero sus argumentos se pueden apoyar en los postulados pedagógicos de la sesión anterior.

Dinámicas Lúdicas, Juegos de Tablero, Juegos Digitales Presenciales, Juegos Virtuales Individuales y Juegos Virtuales Colaborativos (Martinez \& Marquis, 2008) ${ }^{5}$

Comunicar por el juego (Advertgame) (Sebastien, 2008) ${ }^{6}$ : juegos publicitarios, Capacitar Jugando (Trainingame): permiten evaluar los conocimientos y ajustar una capacitación en función del nivel de cada uno de los individuos, Juegos que Ejercitan el Cerebro (Brain Games): juegos que ayudan a desarrollar la lógica

5 Javier Martínez Aldanondo, director de la consultora "Juego Serio".

6 Algunas son tomadas de SoyCulto: Agencia de medios inteligentes. 
y el razonamiento, ejemplo de estos, tenemos: Ajedrez, Sudoku y Reversi.

Simulaciones: juegos que emulan la realidad (Navarro, 2004), i.e. simuladores de vuelo, como los de Flight Simulator, SimCity, Army, Battlezone, y también en el uso militar (Carbonel, 2008).

Juegos de Rol: los participantes asumen el papel de los personajes del juego en un mundo ficticio, ayudados por un narrador ${ }^{7}$,e. g. El Señor de los Anillos, Calabozos y Dragones, Entrevista con el Vampiro, Matrix, StarWars, Battlestar Galactica, etc. A partir de los juegos de rol aparecen los videojuegos de rol, comúnmente conocido como RPG (siglas en inglés de role playing game) que requieren del computador para ser jugados.

\section{F. Niveles de APLiCACIón dE LOS JUEgos}

Del artículo Feature Serious Games (Alhadeff, 2008) ${ }^{8}$ se pueden extraer los siguientes elementos que nos permiten su aplicación en el contexto de la enseñanza de la ingeniería del software a través de juegos: inicialmente la definición de "juego" de Fetcher (Fetcher, 1971) ${ }^{9}$.

Los juegos pueden tener tres niveles de aplicación:

7 Wizards Of The Coast es una empresa estadounidense fundada por Peter Atkinson dedicada a los juegos. Subsidiaria de Hasbro. Su mayor aportación son los juegos Magic: El Encuentro y DungeonDungeon (D\&D). También crearon un juego de cartas sobre el mundo mágico de Harry Potter.

8 http://futuremakingseriousgames.com/

$9 \quad$ El juego.se define de acuerdo a las siguientes características:

1. Hay un conjunto de jugadores (dos o más).

2. Hay un conjunto de reglas que proporcionan pautas de comportamiento para los jugadores.

3. El conjunto de posibles resultados esta especificado o determinado.

4. Hay un conflicto de intereses entre los jugadores.

5. Cada jugador tiene una cierta capacidad de actuación (un conjunto de recursos) y un modelo de preferencias entre las metas.

6. Hay un sistema de información 
- Pre-instruccional: a través de los juegos el estudiante puede llegar a descubrir por sí mismo el concepto o hallar una justificación de lo que se pretende dar a conocer; por lo tanto el juego puede ser una guía en el aprendizaje del estudiante.

- Co-instruccional: el juego puede ser una de las herramientas utilizadas por el docente para apoyar sus actividades de enseñanza.

- Post-instruccional: el juego se puede tomar como una actividad para reforzar, evaluar y consolidar el aprendizaje de un tema ya enseñado

Estas nivelaciones pueden ser estandarizadas de manera que los juegos siempre se les puedan definir el nivel a cual pertenecen.

\section{Conclusiones}

En este artículo se plantearon postulados pedagógicos que sustentan la clasificación y el uso de los juegos serios en la enseñanza con lo cual se logró identificar que muchos de los juegos que actualmente se utilizan carecen de soportes pedagógicos y otros se pueden soportar en los postulados propuestos.

En el levantamiento del estado del arte de los juegos serios que se levantó, se logró identificar que en muchos juegos hace falta mayor rigor en el desarrollo de estos que garantice la calidad del aprendizaje. Con respecto a los juegos dirigidos específicamente a la enseñanza de la ingeniería del software se encontraron pocos desarrollos pero la mayoría bien sustentados y elaborados con gran rigurosidad.

También se encontró que las TIC permiten un mayor acercamiento al conocimiento, en particular los juegos serios de una manera agradable y motivadora, pero hace falta mayor rigor pedagógico que garantice la calidad del aprendizaje.

En la investigación se encontraron diferentes clasificaciones y formas de utilización de los juegos que sirvió para identificar la carencia de un comité rector y de estándares para el desarrollo de juegos serios. 


\section{VI.Trabajos Futuros}

Definir desde la pedagogía delineamientos que encaminen los juegos a un aprendizaje significativo, tomar los juegos actuales y realizar evaluaciones pedagógicas que permitan su reconstrucción.

Continuar con la creación de estándares para los juegos serios, al igual que la creación de un comité internacional que los supervise y proyecte.

Se deben de investigar los juegos serios que funcionan en diferentes áreas del saber con el fin de adaptarlos a la enseñanza y aprendizajes de temas de ingeniería del software teniendo en cuenta el Modelo para la Enseñanza de la Ingeniería del Software SwEBor de la IEEE.

Continuar con el desarrollo de juegos serios soportados pedagógicamente y didácticamente.

\section{Agradecimientos}

Mis más sinceros agradecimientos a los diferentes autores e investigadores sobre juegos serios, igualmente a los desarrolladores de juegos que han permitido una gran evolución en los procesos de enseñanza aprendizaje y en la utilización de las TICs en la educación.

Agradezco, a los estudiantes integrantes del semillero de investigación en desarrollo de software 2008 del ITM, por su colaboración, apoyo y tenacidad en la realización de las tareas propuestas que permitieron un mejor resultado de este trabajo.

Agradezco la colaboración del PhD Edilson Delgado Trejos del Centro de Investigaciones del ITM por su asesoría y apoyo en la investigación.

Esta investigación es el resultado de acciones concretas que se vienen desarrollando en la Jefatura de Sistemas de Información, bajo la jefatura de Fray León Osorio Rivera del Instituto Tecnológico Metropolitano - ITM, para fortalecer el perfil del 
ingeniero de Sistemas, como desarrollador de software. Al igual que es un resultado del trabajo de grado de la Maestría en Ingeniería Informática de la Universidad Eafit que está llevando a cabo el profesor Diego Alejandro Guerrero Peña bajo la asesoría del Phd. John Antonio Trujillo Vargas Profesor titular de la universidad Eafit y la Phd Raquel Analya Profesor titular de la universidad Eafit.

\section{Referencias}

Alhadeff, Eliane. Future-Making Serious Games Blog explores how games can be used for building a better future. [En línea] [Citado 18 de septiembre de 2008]. Disponible en Internet http://elianealhadeff.blogspot. com/ y http://futuremakingseriousgames.com/

Anaya, Raquel (2006). Una visión de la enseñanza de la ingeniería de software como apoyo a las empresas de software. Medellín: Revista Universidad Eafit. ISSN 0120-033X. Vol. 042 Número: 0141 Año: 2006

Anaya, Raquel y Trujillo, John. (2006),”Un modelo para la enseñanza en contexto de la Ingeniería del Software". Medellín: Departamento de Informática y Sistemas de la Universidad Eafit. Medellín: Revista Universidad Eafit. ISSN 0120-033X

Bass, Jossey. (1999). La Enseñanza para la comprensión (Título original: The Teaching for Understanding Guide).ED Paidos. ISBN 950-12-55026.

Bierre. K. and Phelps, A. (2004) The Use of M.U.P.P.E.T.S. in an Introductory Java Programming Course, In Proc. of the 5th Annual SIGITE Conference, 122-127.

Blázquez, Daniel, EXEBlog desarrollo de videojuego y más. [En línea] [Citado en 13 de octubre de 2008]. Disponible en Internet: http://www.exelweiss.com/blog/

Boiffard . Mushromm Corporation. Wellcome to Warp Zone. [En línea] [Citado 4 de octubre de 2008]. Disponible en Internet: http://www.mushroomcorporation.com. A. (2008A) Global Conflict: Palestine [En línea] [Citado 28 de agosto de 2008]. Disponible en Internet: http://www.mushroomcorporation.com/ . 
Bordergames. Lavapies. [En línea] [Citado 7 de octubre de 2008]. Disponible en Internet: http://www.sindominio.net/fiambrera/bordergames/index. htm

Carless, S. Informa predicts $\$ 58.4 \mathrm{~B}$ game industry in 2007. Gamasutra (Oct.24,2005); www.gamasutra.com/php-bin/news_index.php?story=6942

Carbonel, Javier. Serious Games: Cuando los juegos tienen otras finalidades. La Cofa Blog de vigilancia tecnológica. Junio 4, 2008. [En línea] [Citado 13 de oct. de 2008]. Disponible en Internet: http://technorati.com/tag/ serious+games

Colon, A. J. (2002).La (de) construcción del conocimiento pedagógico: Nuevas perspectivas en la Teoría de la educación “. Barcelona: Ediciones Paidos, (Pág. 83-157).

Duvall, R., Chotin, Neuringer, M., Goldberg, D., and DVAN, A. Object-Oriented Programming Chapters (draft): Online: http://www.cs.brown.edu/ courses/cs015/2001/Chapters/contents.html

Eger, Chrustopher, Bierre, Kevins, Phelps, Andrew y Ventura Phil, "Hello, M.U.P.P.E.T.S.: Using 3D Collaborative Virtual Environment to Motivate Fundamental Object-Oriented Learning”, Revista OOPSLA'06; ACM 1-59593-491-X/06/0010, U.S. October 22-26, 2006, (draft): Online: http://www.cs.brown.edu/courses/cs015/2001/Chapters/contents.html

Feature Making. Serious Games. [En línea] [Citado 18 de octubre de 2008]. Disponible en Internet: http://futuremakingseriousgames.com/

Fetcher, J, L. "The effectiveness of simulation game as learning environments.”. Revista: Simulation and Games, vol 2, 1971, pp 425-454.

Gairin. José Maria. (1990). "Efectos de la utilización de juegos educativos en la enseñanza de las matemáticas”. Revista: Educar, Zaragoza, ISSN 0211-819X, Universidad Autónoma de Barcelona, vol 17, pp. 105-118.

Guerrero. Diego Alejandro. (2007). Revista Tecnológicas "Estrategias Didácticas para la Ingeniería del Software a partir del proyecto SWEBOK”. Revista Tecnológicas Nº 18 ISSN 0123-7799, Medellín. pp. 185-224.

Houck, Mark; Ibraim,Zia; Serious Games: A place for people with an interest in serious games. [En línea] [Citado en 13 de septiembre de 2008]. Disponible en Internet:http://seriousgames.ning.com/

Intelligent Windows Management. Turn It All Off. [En línea] [Citado 20 de octubre de 2008]. Disponible en Internet: http://www.turnitalloff.com/ play.htm 
Leontiev, A. N. (1981). Problems of the development of the mind. Moscow: Editorial Progress..

Martinez, Javier; Marquis, Lucio. "Divertirse para aprender:¿Una regla de oro?.”. Revista: Learning Review latinoamérica, año 5, № 23, abrilmayo-junio 2008. pp 18-22. [En línea] [Citado 17 de septiembre de 2008]. Disponible en Internet: http://www.learningreview.com/juegos-seriospara-el-aprendizaje/articulos-y-entrevistas/divertirse-para-aprenderuna-regla-de-oro--1099-2.html

Navarro. E and Van. A. (2004) "SimSE: An Educational simulation game for teaching the software engeneering process", Proceeding of the 9th annual SIGCSE conference on Innovation and technology computer science education, Leeds. pp. 233-233

Nintendo. Nintendo Entertainment System: [En línea] [Citado 28 de octubre de 2008]. Disponible en Internet: http://es.wikipedia.org/wiki/NINTENDO_Entertainment_System

Peshette, Alix y Thornburg, David, “Can Games Be Used To Teach”. (2006) Revista Learning \& Leading with Technology; ISTE( International Society for Technology in Education), ISSN 1.800.336.5191, U.S. \& Canada, vol 7, abril, [En línea] [Citado en 13 de septiembre. de 2008]. Disponible en Internet: http://www.eduteka.org/SiNoJuegosEducacion.php

Phelps, A., Bierre, K., and Parks, D. (2003). M.U.P.P.E.T.S: Multiuser Programming Pedagogy for Enhancing Traditional Study, In Proc. of the 4th CITC Conference, 100-105

Pozo, J.I. (1989) “Teorías cognitivas del aprendizaje”. Madrid: Ed. Morata.

Pressman, Roger.(2000). Software Engineering: A Practitioner's Approach, $6^{\circ}$ Ed. McGraw-Hill, New York.

Sebastien Yanni. SoyCulto: Agencia de medios inteligentes. [En línea] [Citado 18 de septiembre de 2008]. Disponible en Internet: http://www. soyculto.com/index.html?framepage=http://www.soyculto.com/Serious_ gaming/Tipos_juegos_serios/Advertgame.html y http://www.soyculto. com/index.html?framepage=http://www.soyculto.com/Serious_gaming/ Tipos_juegos_serios/Trainingame.html

Trujillo V, John Antonio y Jaramillo R., Carlos Mario, "Estrategias didácticas en educación superior con la mediación de la computación móvil”, Revista: Educación y Pedagogía, Medellín, Universidad de Antioquia, facultad de Educación, vol. XVIII, num. 45, (Mayo-agosto), 2006, pp. 93107. 
Under Ash. Under Siege. [En línea] [Citado 7 de octubre de 2008]. Disponible en Internet: http://www.afkarmedia.com y http://www.underash.net/

Unreal Technology. 9-11 SURVIVOR. [En línea] [Citado 7 de octubre de 2008]. Disponible en Internet: http://www.selectparks.net/archive/ 911survivor/

U.S. Office of Management and Budget. Historical Tables (Table 3.1.Outlays by Superfunction and Function: 1940-2009). In Budget of the U.S. Government, Fiscal Year 2005, Washington, D.C., 2004, 45-52; www. whitehouse.gov/omb/budget/fy2005/pdf/hist.pdf.

Urrego, María Idilia; Castaño, Luz Elena. Modelo pedagógico. Medellín: Instituto Tecnológico Metropolitano. 1999.

Vega, José Ramón. Pervasive Games: juegos sin límite [En línea] [Citado 28 de octubre de 2008]. Disponible en Internet: http://www.luciomargulis. com.ar/?p\&paged $=5$

Vygotski, L. S. (1996). El desarrollo de los procesos psicológicos superiores. Barcelona: Crítica traducción de "Mind in Society. The development of Higher Psychological Processes" Cambridge, Massachusetts: Harvard University Press.

Ward, Darrell. E. Instructions, Simples Solutions. Real Solutions. [En línea] [Citado 18 de septiembre de 2008]. Disponible en Internet: http://www. einstruction.com/

Zapata, Carlos Mario, "Un Curso Inicial de Ingeniería del Software basado en Juegos", Revista: Tendencias en ingeniería de software e inteligencia artificial, Medellín, LitoNueve Universidad Nacional de Colombia, ISBN 978-958-44-1344-4, 2007, pp. 17-23.

Zyda, Michael. "Creating a Science of Games," Revista: Communications of the ACM, Vol. 50, Julio 2007, No. 7, pp 27- 29. [En línea] [Citado 15 de octubre de 2008]. Disponible en Internet: http://gamepipe.usc.edu/ zyda/ pubs/CACM-July2007.pdf 